\title{
A Semi-Automated Workflow for Segmenting Contents of Single Cardiac Cells from Serial-Block-Face Scanning Electron Microscopy Data
}

\author{
$\underline{\text { Akter Hussain }}^{1}$, Eric Hanssen ${ }^{2}$ and Vijay Rajagopal ${ }^{1,3}$ \\ 1. Cell Structure and Mechanobiology Group, Dept. of Mechanical Engineering, University of \\ Melbourne, Melbourne, Australia. \\ 2. Advanced Microscopy Facility at Bio21 Molecular Science and Biotechnology Institute, University of \\ Melbourne, Melbourne, Australia. \\ 3. Systems Biology Laboratory, Melbourne School of Engineering, University of Melbourne, Australia.
}

Serial-block-face scanning electron microscopy (SBFSEM) has revolutionized electron microscopy of cellular ultrastructure by providing a high throughput method to image large fields of view at tens of nanometer $(\mathrm{nm})$ resolution. These data are enabling scientists to visualize and understand the intricate details of cellular architecture in health and disease. For examples within cardiac physiology, SBFSEM has enabled scientists to visualize the distribution of mitochondria in health and heart failure ${ }^{1}$ and examine the distribution of the sarcoplasmic reticulum ${ }^{2}$.

These data are also rich with information that can be used to develop computational models of the cell for investigations into the mechanisms that link the structural organization of the cell to its function. Current models of cardiac cell structure have either been derived from confocal microscopy ${ }^{3}$, which provides much lower resolution than SBFSEM, or they are derived from electron tomography data ${ }^{4}$, which provide high resolution but very small fields of view in comparison to SBFSEM. The challenge of electron microscopy data is the low contrast in the data that makes rapid automatic segmentation and model creation extremely challenging, such that current models derived from these images typically involve tedious manual segmentation of the components of the cell. There is currently no published method for segmentation of information pertaining to a single cell. We are developing methods to enable rapid creation of structurally detailed computational models from SBFSEM data. Our aim is to develop computational models that are at high resolution but also span the entire domain of a single cell ( $\sim 20$ micrometer diameter, 100 micrometer length). This paper presents a novel algorithm that we have developed to semi-automatically segment the contents of an entire heart cell from SBFSEM data.

Animal procedures followed guidelines approved by the University of Auckland Animal Ethics Committee (for animal procedures conducted in Auckland, Application Number R826). Tissue blocks were prepared for SBFSEM from the left ventricular wall of a sixteen-week old male Sprague Dawley rat A sixteen-week old male as detailed in ${ }^{5}$. A block was chosen for SBFSEM and segmentation for this paper. The block was trimmed to a square block face of $1 \mathrm{~mm}^{2}$ and $300 \mu \mathrm{m}$ deep with fibers running perpendicular to the future cutting face. The sample was mounted on a Teneo VolumeScope (FEI, Hillsborough, USA) and imaged in low vacuum mode (50 mbar) with the VolumeScope backscattered detector at $3 \mathrm{kV}$ with a specimen current of $0.1 \mathrm{nA}$ and a pixel size of $10 \mathrm{~nm}$. Four thousand and nineteen serial sections of $50 \mathrm{~nm}$ in thickness were acquired. The sections were align using IMOD ${ }^{6}$, The section were then rotate 90 degrees around the $\mathrm{Y}$ axis and the data binned to an isotropic voxel size of $50 \mathrm{x} 50$ $\mathrm{x} 50 \mathrm{~nm}$. This dataset is one of the largest that has been collected of the cardiac cell at this resolution.

The segmentation algorithm requires as input, contours of the cell boundary that have been manually segmented by the user at selected image planes within the dataset. We manually segmented every $10^{\text {th }}$ 
image along the longitudinal axis of the cell using IMOD from 571 slices that contained the cell that we targeted for segmentation. A region based thresholding algorithm, which automatically identified a threshold (with no user input), was then applied to these manually segmented regions. The algorithm split the pixels into mitochondria and myofibril groups. We input manual contours for the nucleus that was then used to extract the nuclear region from the data as well. Existing segmentations were propagated to un-segmented slices iteratively and refined to extract the entire segmented dataset. An important step in our refinement procedure involved applying the region-based thresholding algorithm to the other viewing axes. This allowed us to check whether a pixel that is classified as mitochondrion (or myofibril) was also classified as such in the other two planes. A pixel is classified into the category for which it has been classified in two out of the three planes. Comparison of the output (Fig.2) with manual segmentations showed that the algorithm had 97\% sensitivity (rate of true positives) and specificity (true negative rate). In further development, we are refining the algorithm to extract other intricate features, such as the membrane networks to develop a complete model of the cardiac cell.

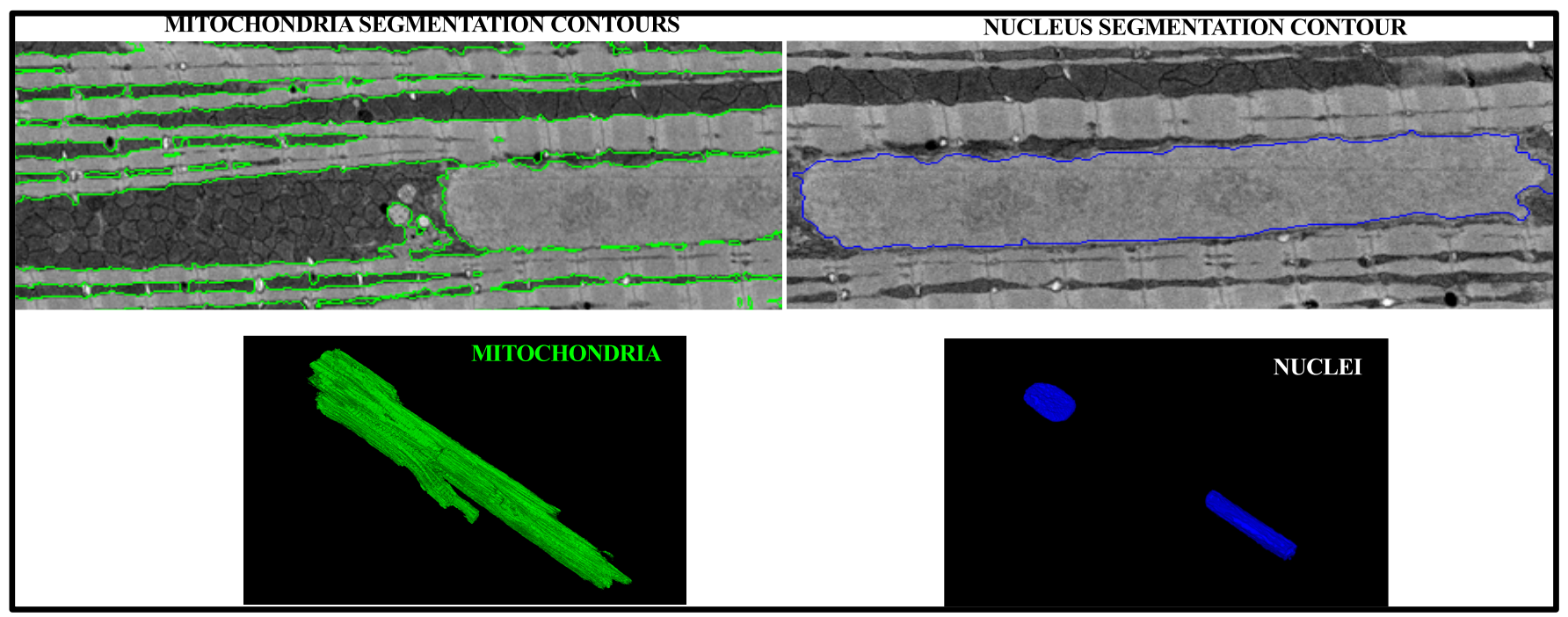

Figure 1: Result of the automatic segmentation of myofibrils, mitochondria and nuclei of a single cell within our SBFSEM dataset

\section{References:}

1. Holzem, K. M. et al. The FASEB journal 30, fj.201500118R-2707 (2016).

2. Pinali, C., et al. Circ Res 113, 1219-1230 (2013).

3. Soeller, C., et al. Exp Physiol 94, 496-508 (2009).

4. Rajagopal, V. et al. PLoS Comp Biol 11, e1004417 (2015).

5. Jarosz, J. et al. AJP: Cell Physiology 312, C190-C197 (2017).

6. Kremer, J. R., Mastronarde, D. N. \& McIntosh, J. R. J Struct Biol 116, 71-76 (1996). 